\title{
Hyperbaric oxygen promotes not only glioblastoma proliferation but also chemosensitization by inhibiting HIF1a/HIF2a-Sox2
}

\author{
Pan Wang ${ }^{1,2}$, Sheng Gong ${ }^{1}$, Jinyu Pan', Junwei Wang ${ }^{1}$, Dewei Zou', Shuanglong Xiong ${ }^{3}$, Lu Zhao', Qian Yan',
} Yangming Deng ${ }^{1}$, Nan Wu $\mathbb{B}^{1}$ and Bin Liao ${ }^{1}$

\begin{abstract}
There exists a consensus that combining hyperbaric oxygen ( $\mathrm{HBO})$ and chemotherapy promotes chemotherapy sensitivity in GBM cells. However, few studies have explored the mechanism involved. HIF1a and HIF2a are the two main molecules that contribute to GBM malignant progression by inhibiting apoptosis or maintaining stemness under hypoxic conditions. Moreover, Sox2, a marker of stemness, also contributes to GBM malignant progression through stemness maintenance or cell cycle arrest. Briefly, HIF1a, HIF2a and Sox2 are highly expressed under hypoxia and contribute to GBM growth and chemoresistance. However, after exposure to HBO for GBM, whether the expression of the above factors is decreased, resulting in chemosensitization, remains unknown. Therefore, we performed a series of studies and determined that the expression of HIF1a, HIF2a and Sox2 was decreased after HBO and that HBO promoted GBM cell proliferation through cell cycle progression, albeit with a decrease in stemness, thus contributing to chemosensitization via the inhibition of HIF1a/HIF2a-Sox2.
\end{abstract}

\section{Introduction}

As the most malignant tumour in the brain, glioblastoma (GBM) is characterized as highly aggressive and infiltrative ${ }^{1}$. Despite advancements in surgical interventions, patients still exhibit a poor prognosis ${ }^{2}$. Even with continuous improvements in chemotherapy, the survival time is extended by only approximately 2 months ${ }^{2,3}$. Previous studies ascribe this effect to the hypoxic microenvironment ${ }^{4}$ and the existence of GBM stem cells $(\mathrm{GSCs})^{5}$, which are thought to be negative and vital factors to patient prognosis. Many approaches have been developed to alleviate the hypoxic environment ${ }^{6}$; however,

\footnotetext{
Correspondence: Nan Wu (wunan881@tmmu.edu.cn) or

Bin Liao (Liaobin@tmmu.edu.cn)

'Department of Neurosurgery, Chongqing General Hospital, University of

Chinese Academy of Sciences, Chongqing 401147, China

${ }^{2}$ Chongqing Medical University, Chongqing, China

Full list of author information is available at the end of the article

These authors contributed equally: Pan Wang, Sheng Gong.

These authors contributed equally: Nan Wu, Bin Liao.

Edited by: Alessandro Rufini
}

the most promising way to address this problem still appears to be hyperbaric oxygen $(\mathrm{HBO})^{6,7}$. Unfortunately, whether HBO alone promotes or inhibits GBM growth remains controversial. Stuhr et al. ${ }^{8}$ showed that tumour growth was inhibited after subcutaneously transplanted GBM cells were exposed to HBO. However, Wang et al. ${ }^{7}$ demonstrated that HBO promoted GBM growth. Therefore, we aimed to address this inconsistency first.

Despite the inconsistent results described above, there is a consensus that combining $\mathrm{HBO}$ and chemotherapy can reduce GBM growth since GBM cells become sensitive to chemotherapy ${ }^{6,9,10}$. However, few studies have explored the mechanism by which HBO promotes chemosensitization to GBM cells. Hypoxic-inducible factor- $1 \alpha$ (HIF1 $\alpha)$ and hypoxic-inducible factor- $2 \alpha$ (HIF $2 \alpha$ ) are the two main molecules that contribute to GBM malignant progression under hypoxic conditions ${ }^{5,11}$. Moreover, Sox2, a marker of stemness, also contributes to GBM malignant progression through stemness maintenance or cell cycle arrest ${ }^{12,13}$. However, after exposure to $\mathrm{HBO}$, whether the

\section{(c) The Author(s) 2021}

(c) (i) Open Access This article is licensed under a Creative Commons Attribution 4.0 International License, which permits use, sharing, adaptation, distribution and reproduction cc) in any medium or format, as long as you give appropriate credit to the original author(s) and the source, provide a link to the Creative Commons license, and indicate if changes were made. The images or other third party material in this article are included in the article's Creative Commons license, unless indicated otherwise in a credit line to the material. If material is not included in the article's Creative Commons license and your intended use is not permitted by statutory regulation or exceeds the permitted use, you will need to obtain permission directly from the copyright holder. To view a copy of this license, visit http://creativecommons.org/licenses/by/4.0/. 
expression of the above factors is decreased, resulting in chemosensitization, remains unknown. Therefore, we performed a series of studies to determine whether the expression of HIF1 $\alpha$, HIF $2 \alpha$ and Sox 2 was decreased after $\mathrm{HBO}$ and whether HBO promoted GBM growth through cell cycle progression but with a decrease in stemness, thus resulting in chemosensitization under the inhibition of HIF $1 \alpha / H I F 2 \alpha-S o x 2$.

\section{Results}

\section{HBO increases tumour volume but promotes} chemosensitization

Magnetic resonance imaging (MRI) revealed larger tumour volumes in mice exposed to HBO than in control mice exposed to normoxia in the absence of TMZ (Fig. 1A, B, D and Supplementary Fig. 1C). In addition, tumour weights increased after $\mathrm{HBO}$ treatment (Fig. $1 \mathrm{C}$ and Supplementary Fig. 1B). Immunohistochemistry (IHC) showed that these tumour tissues highly expressed Ki67 and $\mathrm{Bcl} 2$ under $\mathrm{HBO}$, but there were no expression of $\mathrm{Ki} 67$ and $\mathrm{Bcl} 2$ in the control group (Supplementary Fig. 1A). Survival analysis showed that the mice that were exposed to $\mathrm{HBO}$ had a short survival time, with a median survival time of approximately 22 days; however, survival was prolonged in control mice, with a median survival time of approximately 26 days (Fig. 1E, F and Supplementary Fig. 1D). Then, the same strategy as described above was used for the other two groups but under TMZ $(2 \mathrm{mg} / \mathrm{kg}$ ) treatment. MRI revealed that after 21 days, tumour volumes and weights decreased significantly in both groups after TMZ treatment; however, the tumour volumes and weights were much smaller in the group that was exposed to $\mathrm{HBO}$ than in the control group (Fig. 1A-D and Supplementary Fig. 1B, C). Then, the survival time of the two groups under the same TMZ treatment was analysed, and the results revealed that mice that were exposed to $\mathrm{HBO}$ had a longer survival time, with a median survival of approximately 37 days, than control mice, with a median survival time of only 30.5 days (Fig. 1E, F and Supplementary Fig. 1D). In addition, the in vitro study revealed a higher proliferative rate of the cells exposed to $\mathrm{HBO}$ than the control cells without $\mathrm{HBO}$ exposure in the absence of TMZ (Fig. 1G and Supplementary Fig. 1E). However, the growth trend of cells exposed to $\mathrm{HBO}$ was lower than that of control cells when both groups were treated with TMZ (Fig. 1G and Supplementary Fig. 1E). In addition, the HBO exposure group had a higher rate of apoptosis than the control group with the same TMZ treatment $(400 \mu \mathrm{M})($ Fig. $1 \mathrm{H})$. Briefly, in the absence of TMZ, HBO exposure increased tumour volume and shortened the survival time; however, after TMZ was added, the group of mice exposed to $\mathrm{HBO}$ had a longer survival time and a smaller tumour volume.

\section{HBO alleviates the hypoxic environment and inhibits both HIF1a and HIF2a}

CGGA database showed that both HIF1 $\alpha$ and HIF $2 \alpha$ were highly expressed in glioma tissues (Fig. 2A). IHC demonstrated that both HIF $1 \alpha$ and HIF $2 \alpha$ were highly expressed in primary GBM tissues (Fig. 2B). Tumour tissues from intracranial transplantation also showed that both HIF $1 \alpha$ and HIF $2 \alpha$ were highly expressed, and these tissues had high expression of hypoxyprobe ${ }^{\mathrm{TM}}-1$ (Fig. 2B). However, the hypoxic environment was alleviated according to hypoxyprobe ${ }^{\mathrm{TM}}-1$ results after $\mathrm{HBO}$ treatment, and both HIF1 $\alpha$ and HIF $2 \alpha$ were decreased significantly after $\mathrm{HBO}$ exposure according to $\mathrm{IHC}$, reverse transcription quantitative PCR (RT-qPCR) and western blot results (Fig. 2B-D). Then, the vitro studies showed that these cells under hypoxic conditions highly expressed hypoxyprobe ${ }^{\mathrm{TM}}-1$, HIF1 $\alpha$ and HIF2 $\alpha$, whereas the cells exposed to $\mathrm{HBO}$ did not express hypoxyprobe ${ }^{\mathrm{TM}}-1$, HIF $1 \alpha$ and HIF2 $\alpha$ (Fig. 2E-G).

\section{Inhibition of HIF1a and HIF2a increases tumour volume but promotes chemosensitization}

We next wondered whether the decreased expression of HIF $1 \alpha$ and HIF $2 \alpha$ under HBO conditions was the reason for cell proliferation and chemosensitization. We first knocked out HIF1 $\alpha$ and HIF2 $\alpha$ in GBM cells and divided them into four groups: empty vector, HIF1 $\alpha-\mathrm{KO}, \mathrm{HIF} 2 \alpha-$ $\mathrm{KO}$ and dual HIF $1 \alpha / \mathrm{HIF} 2 \alpha-\mathrm{KO}$ (Supplementary Fig. 2A). We implanted these cells into mouse brains and found that the tumour volumes and weights of the HIF $1 \alpha$-ko or HIF2 $\alpha$-ko individually group were lower than those in the empty vector control group, but the tumour volumes of the dual HIF1 $\alpha$ and HIF2 $\alpha$ knockout group were significantly large, with the largest tumour weight and shortest survival time among all the groups (Fig. 3A-E). IHC showed that the tumour tissues of the HIF1 $\alpha$-ko or HIF2 $\alpha$-ko individual group had lower expression of Ki67 and Bcl 2 than the control and the dual HIF $1 \alpha$ and HIF $2 \alpha$ knockout group (Supplementary Fig. 2B). After the intraperitoneal injection of TMZ $(2 \mathrm{mg} / \mathrm{kg})$ into the above groups, the tumour volume decreased after HIF1 $\alpha$ or HIF $2 \alpha$ knockout, and the smallest tumour volume was observed in the group with simultaneous HIF1 $\alpha$ and HIF $2 \alpha$ knockout. In addition, after TMZ treatment, the group with both HIF $1 \alpha$ and HIF $2 \alpha$ knockout had the lowest tumour weight and longest survival time among all groups (Fig. 3A-E). Then, the cells described above were cultured in $1 \% \mathrm{O}_{2}$ for $72 \mathrm{~h}$ in the absence of $\mathrm{TMZ}$, and there were no significant differences in cell proliferation between HIF1 $\alpha-\mathrm{KO}$ cells, HIF $2 \alpha-\mathrm{KO}$ cells and control empty vector cells. However, cell proliferation increased after the simultaneous knockout of HIF1 $\alpha$ and HIF $2 \alpha$ compared with the empty vector, HIF $1 \alpha-\mathrm{KO}$ and HIF $2 \alpha-$ KO cells (Fig. 3F and Supplementary Fig. 2C). Then, we 


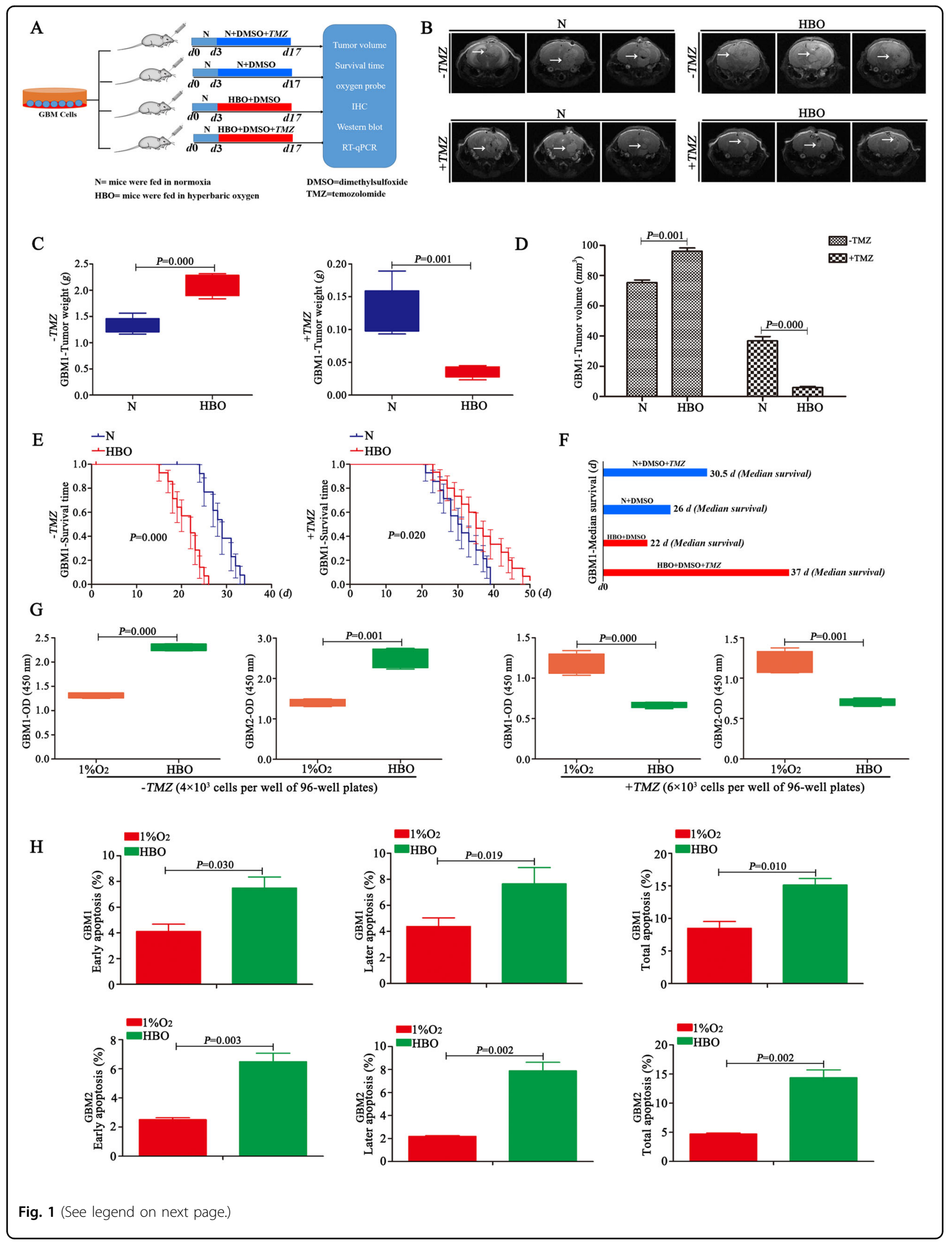


(see figure on previous page)

Fig. 1 HBO increases tumour volume but promotes chemosensitization. A Schematic process of the in vivo study with or without HBO treatment. B-D MRI showed that the tumour volume was larger and the tumour weight was higher in the group under $\mathrm{HBO}$ conditions than in the control group without HBO treatment in the absence of TMZ. However, after treatment with the same dose of TMZ ( $2 \mathrm{mg} / \mathrm{kg}$ ), the tumour volume decreased and the tumour weight was decreased in the group exposed to HBO compared with the control group. $\mathbf{E}, \mathbf{F}$ In the absence of TMZ, the survival time became shorter after $\mathrm{HBO}$ exposure (median survival time of the HBO treatment group vs control group $=22$ vs 26 days). However, after TMZ treatment $(2 \mathrm{mg} / \mathrm{kg})$, the group that was exposed to HBO had a much longer survival time than the control group without HBO treatment (median survival of the $\mathrm{HBO}$ treatment group vs control group $=37$ days vs 30.5 days). $\mathbf{G}$ The cells exposed to HBO had a higher proliferation rate than the control cells without $\mathrm{HBO}$ treatment in the absence of TMZ, but the cells exposed to HBO had a lower growth trend than the control cells without $\mathrm{HBO}$ following TMZ treatment $(400 \mu \mathrm{M})$. $\mathbf{H}$ The HBO treatment group had a higher apoptosis rate than the control group with the same TMZ treatment $(400 \mu \mathrm{M})$. The $P$ value was determined by an independent samples $t$-test, and the survival time was analysed by the log-rank test.

added TMZ $(400 \mu \mathrm{M})$ to the culture medium, and the results revealed increased apoptosis after HIF $1 \alpha$ or HIF $2 \alpha$ knockout, and the highest apoptosis rate was observed in dual HIF $1 \alpha$ and HIF $2 \alpha$ knockout cells (Fig. $3 \mathrm{H}$ and Supplementary Fig. 2C). As a result, the proliferation rate of HIF $1 \alpha-K O$ or HIF $2 \alpha-K O$ cells decreased, and dual HIF1 $\alpha$ and HIF2 $\alpha$ knockout cells exhibited the lowest growth rate after the same TMZ treatment $(400 \mu \mathrm{M})$ (Fig. 3G).

\section{Sox2 is highly expressed in GBM, and both HIF1a and HIF $2 a$ regulate Sox 2 expression}

CGGA database showed that Sox2 was highly expressed in GBM tissues (Fig. 4A). To compare the expression of Sox 2 in the absence or presence of $\mathrm{HBO}$, we collected tumour tissues from primary GBM and tumour implantation from mouse brains and observed Sox2 was expression in the group without $\mathrm{HBO}$ treatment; however, after HBO treatment, Sox2 expression decreased significantly (Fig. 4B). Then, the in vitro study demonstrated that Sox2 was highly expressed in the cells that were cultured in $1 \% \mathrm{O}_{2}$, but after exposure to $\mathrm{HBO}$, the Sox2 levels decreased significantly (Fig. 4C). RT-qPCR and western blot results were consistent: Sox 2 expression was high in cells that were cultured in $1 \% \mathrm{O}_{2}$ but decreased significantly after $\mathrm{HBO}$ treatment (Fig. 4D, E). Then, we found that both HIF $1 \alpha$ and HIF2 $\alpha$ had a positive correlation with Sox 2 according to analysis of the CGGA database (Fig. 4F). Therefore, we cultured empty vector cells, HIF $1 \alpha-K O$ cells, HIF $2 \alpha-K O$ cells and HIF $1 \alpha / H I F 2 \alpha-$ $\mathrm{KO}$ cells under hypoxic conditions and detected Sox2 expression was lower in HIF1 $\alpha-\mathrm{KO}$ cells and HIF2 $\alpha-\mathrm{KO}$ cells than in vector control cells, and Sox 2 was the lowest in the cells in which both HIF1 $\alpha$ and HIF2 $\alpha$ were knocked out (Fig. 4G).

\section{Stemness markers are highly expressed in GBM, and HBO decreases their expression}

CGGA database and IHC showed high expression of stemness markers, such as CD133, CD9 and Nestin, in GBM (Fig. 5A, B). Then, IHC, RT-qPCR and western blotting were used to detect CD133, CD9 and Nestin in the absence or presence of $\mathrm{HBO}$ in the tumour tissues of mice following intracranial transplantation, and the results showed weak expression of CD133, CD9 and Nestin after HBO treatment. In contrast, if the mice were not exposed to HBO, high expression of CD133, CD9 and Nestin was observed (Fig. 5B-D). The in vitro study yielded similar results: after $\mathrm{HBO}$ exposure, the expression of CD133, CD9 and Nestin was lower than that in the control group without $\mathrm{HBO}$ treatment (Fig. 5E-G).

\section{HBO increases tumour volume but promotes chemosensitization due to cell cycle progression and decreases stemness by inhibiting HIF1a/HIF2a-Sox2}

GBM cells were cultured in $1 \% \mathrm{O}_{2}$, and then one group of cells was maintained in $1 \% \mathrm{O}_{2}$ while the other group was exposed to HBO. The results showed GBM cells after HBO exposure had a low proportion of cells in the $G_{1}$ phase and a high proportion of cells in $\mathrm{G}_{2} / \mathrm{M}+\mathrm{S}$ phase (Fig. 6A). We wondered whether these results were due to the decreased expression of HIF $1 \alpha$ and HIF $2 \alpha$. And then we found a decrease in the proportion of cells in the $G_{1}$ phase and an increase in the proportion of cells in the $\mathrm{G}_{2} /$ $\mathrm{M}+\mathrm{S}$ phase after HIF $1 \alpha$ or HIF $2 \alpha$ knockout individually; however, the change in the cell cycle in the above groups was not statistically significant. Nevertheless, the proportion of cells in the $G_{1}$ phase was significantly lower and the proportion of cells in the $G_{2} / M+S$ phase was significantly higher in the dual HIF1 $\alpha / H I F 2 \alpha-$ ko group than in the other three groups (Fig. 6B). Because Sox2 is regulated by HIF $1 \alpha$ and HIF $2 \alpha$, we investigated changes in the cell cycle after Sox 2 knockout. The results showed no significant differences between the control and empty vector groups; however, the proportion of cells in the $G_{1}$ phase decreased and the proportion of cells in the $G_{2} / M$ $+S$ phase increased after Sox2 knockout (Fig. 6C). We investigated the influence of cell cycle changes on proliferation and found that after Sox 2 knockout, the proliferation rate increased (compared to the control and empty vector) (Fig. 6D). Based on the expression of stemness markers in GBM cells and the upregulation of stemness by HIF $1 \alpha$ and HIF $2 \alpha$, we wondered whether the decrease in stemness resulted from the decrease in Sox2, thus leading to chemosensitization. Therefore, we used 


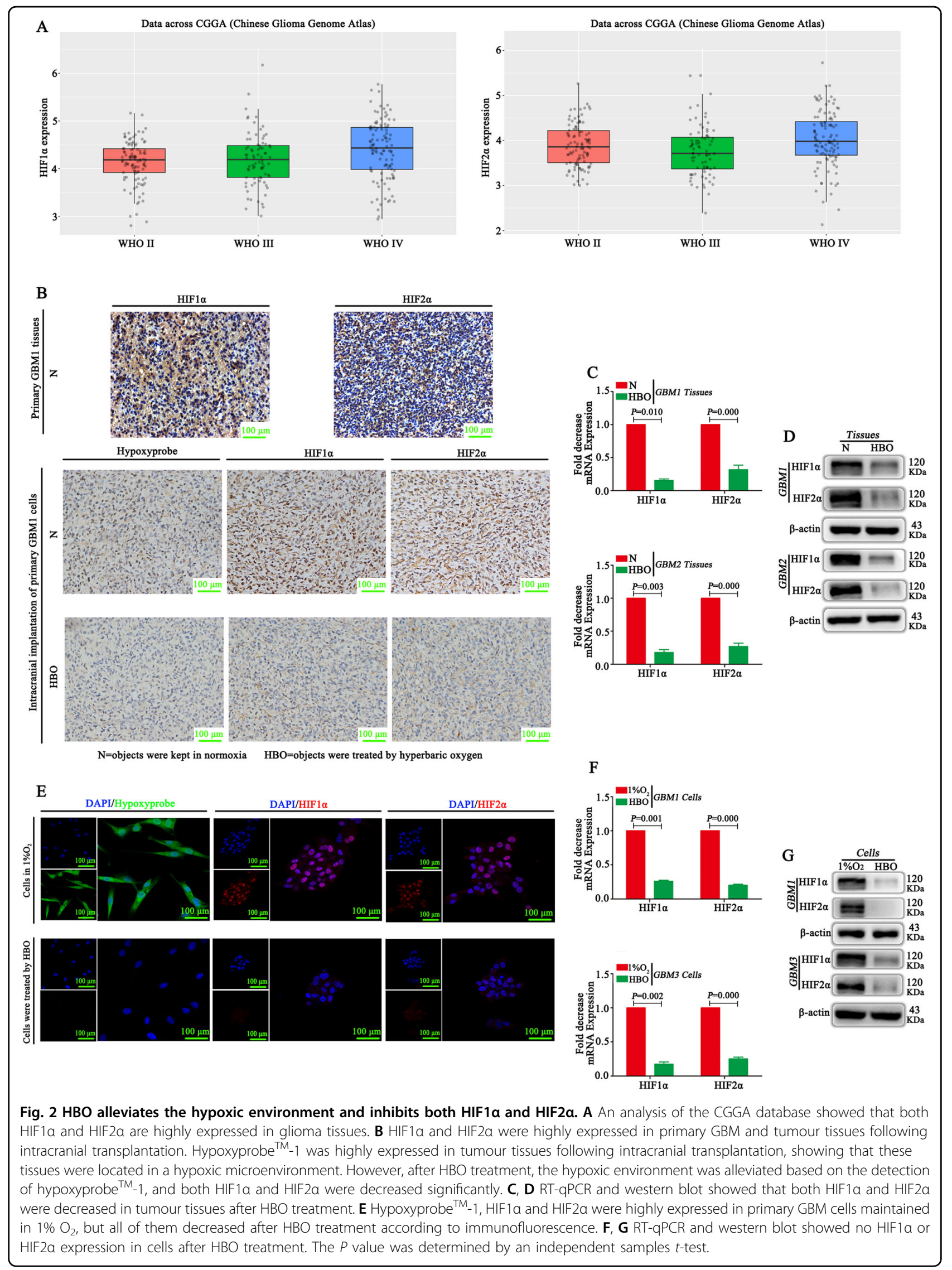




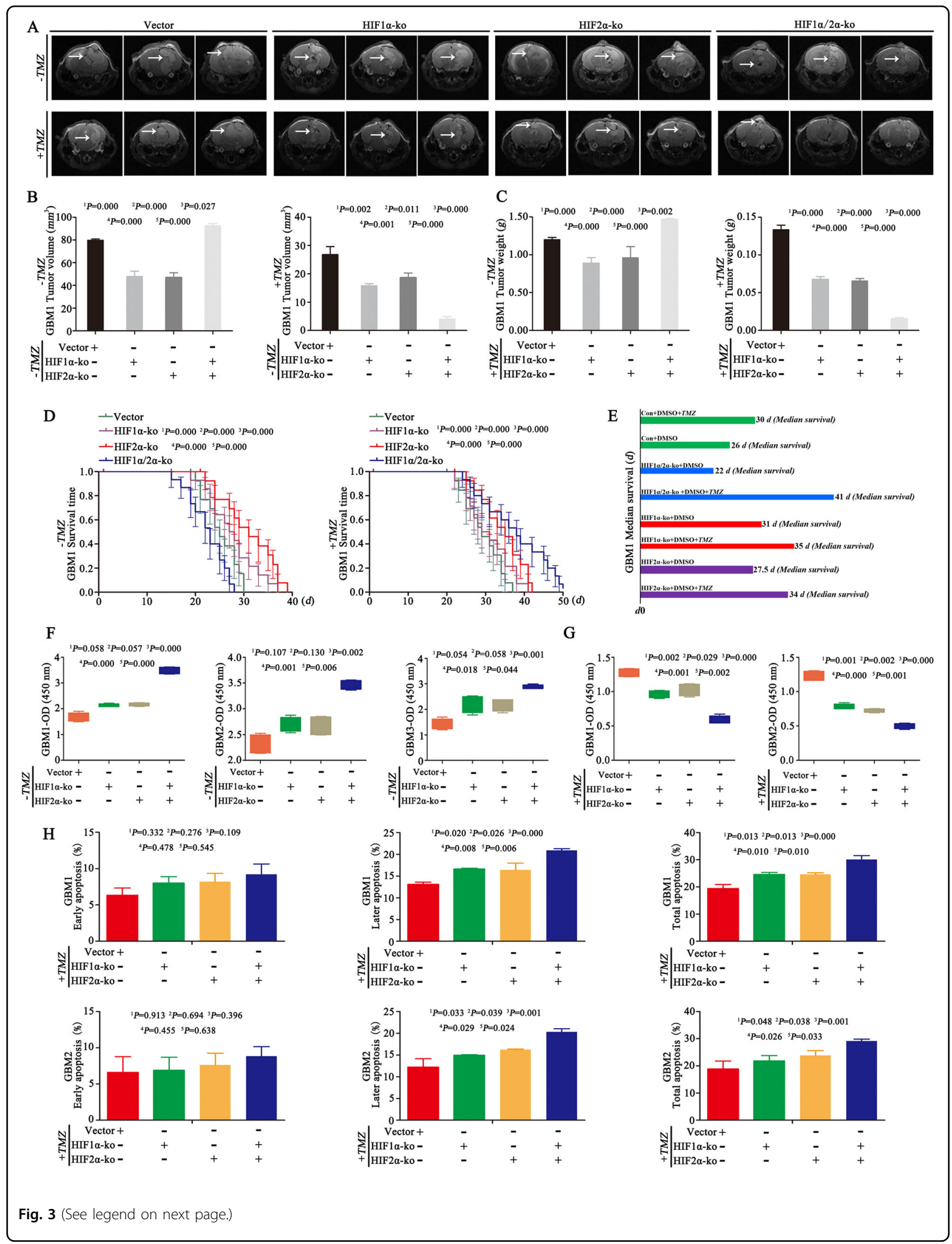


(see figure on previous page)

Fig. 3 Inhibition of HIF1a and HIF2a increases tumour volume but promotes chemosensitization. A-C In the absence of TMZ, tumour volume and weight were decreased after HIF1a or HIF2a-KO individually compared with the empty vector control, but the dual HIF1a and HIF2a knockout group had a larger tumour volume and tumour weight than the other three groups. After TMZ treatment ( $2 \mathrm{mg} / \mathrm{kg})$, the tumour volume decreased after HIF1a and HIF2a knockout individually or simultaneously, and the smallest tumour volume was observed in the group with dual HIF1a and HIF2a knockout. In addition, the group with dual HIF1a and HIF2a knockout had the lowest tumour weight among all the groups. D, E In the absence of TMZ, the survival time became longer after HIF1a or HIF2a knockout individually, but the survival time was shorter in the dual HIF1a and HIF2a knockout group than in the other three groups (median survival time of the empty vector group vs HIF1a-ko vs HIF2a-ko vs HIF1a/HIF2a-ko group $=26$ vs 31 vs 27.5 vs 22 days). However, after TMZ treatment ( $2 \mathrm{mg} / \mathrm{kg})$, the HIF1a or HIF2a knockout group experienced prolonged survival, and the longest survival time was observed in the group with dual HIF1a and HIF2a knockout (median survival time of the empty vector group vs HIF1a-ko vs HIF2a-ko vs HIF1a/HIF2a-ko group $=30$ vs 35 vs 34 vs 41 days). F The above cells were cultured in $1 \% \mathrm{O}_{2}$ for $72 \mathrm{~h}$ in the absence of TMZ, and there were no significant differences in cell proliferation between the HIF1a-KO, HIF2a-KO and control empty vector groups. However, after knocking out both HIF1a and HIF2a, the growth rate became the highest among all the groups. G The above cells were cultured in $1 \% \mathrm{O}_{2}$ for $72 \mathrm{~h}$, and TMZ $(400 \mu \mathrm{M})$ was added to the culture medium for an additional $72 \mathrm{~h}$. After HIF1a or HIF2a was knocked out individually, cell proliferation decreased. After both HIF1a and HIF2a were knocked out, the growth rate became the lowest among all the groups. $\mathbf{H}$ The above cells were cultured in $1 \% \mathrm{O}_{2}$ for $72 \mathrm{~h}$, and TMZ $(400 \mu \mathrm{M})$ was added to the culture medium for an additional $72 \mathrm{~h}$. The apoptosis rate increased after HIF1a or HIF2a knockout individually. After knocking out both HIF1a and HIF2a, the apoptosis rate became the highest among all the groups. The $P$ value was determined by one-way ANOVA, and the survival time was analysed by the log-rank test.

the CGGA database and found a positive correlation between Sox2 and CD133, CD9 and Nestin (Fig. 6E). In addition, we cultured control, empty vector and Sox2-ko cells under hypoxia and found that CD133, CD9 and Nestin levels decreased significantly after Sox2 knockout (Fig. 6F). Next, to verify chemosensitization, we detected cell apoptosis, and the results showed a much higher apoptosis rate in Sox2-ko cells than in control cells (Fig. $6 G)$.

\section{Discussion}

Both the hypoxic environment and GSCs contribute greatly to GBM malignant progression ${ }^{4,5}$. Regarding the correlation between hypoxia and GSCs, previous studies have shown that the hypoxic environment maintains or induces stemness ${ }^{4,14}$, leading to GBM malignant progression mainly due to resistance to radiochemotherapy ${ }^{15}$. Therefore, hypoxia plays an important role, and it is urgent to find ways to alleviate hypoxia. Previous studies have described many ways, such as increasing the ability of red blood cells to carry oxygen or gene therapy to correct the hypoxic environment ${ }^{6}$. However, at present, the most effective way to alleviate hypoxia according to studies is $\mathrm{HBO}^{6,9}$. Unfortunately, to date, only a few studies have examined the influence of $\mathrm{HBO}$ alone on GBM, and there is no consensus on the issues discussed above. In 2015, Wang et al. ${ }^{7}$ performed a series of experiments and found that $\mathrm{HBO}$ promoted malignant glioma growth, thus leading to a poor prognosis. Other researchers reported the same results later ${ }^{9,16}$. In addition, our study demonstrated the formation of large tumours after continuous HBO treatment in vivo; these mice had a relatively shorter survival time than the control mice. However, another study from Stuhr et al. ${ }^{8}$ showed that the tumour volume of subcutaneously transplanted gliomas in C57Bl/6J mice was much smaller after $\mathrm{HBO}$ treatment than in control mice. Here, we find that subcutaneously transplanted glioma cells differ from intracranial glioma cells, and there is no doubt that the results from intracranial gliomas are better than those from subcutaneously transplanted gliomas.

Although several studies have suggested that HBO promotes GBM growth, no reports have described the detailed mechanism. According to the theory that hypoxia inhibits cell cycle progression and arrests the cell cycle in the $\mathrm{G}_{1}$ phase ${ }^{14,17}$, we focused on the influence of $\mathrm{HBO}$ on the cell cycle. Through a series of experiments, we found that the cell cycle progressed, with a high proportion of cells entering the $G_{2} / M+S$ phase after $\mathrm{HBO}$ treatment, thus leading to GBM cell proliferation and increasing tumour volume. In addition, our results revealed that the tissues or cells after HBO had a higher expression of Ki67 and $\mathrm{Bcl} 2$, which means that $\mathrm{HBO}$ promotes cell proliferation. However, the cells under HBO with TMZ treatment had a higher apoptosis rate than the control without HBO but with the same dose of TMZ, which means that $\mathrm{HBO}$ promotes chemosensitization. Next, we wondered why the tumours formed following HBO exposure were sensitive to chemotherapy. Hypoxia maintains the stemness of GSCs and even induces the formation of GSCs from differentiated glioma cells ${ }^{5,18}$. Briefly, stemness markers are highly expressed under hypoxic environments, which is why GBM cells are resistant to chemotherapy. Therefore, we detected stemness markers, including CD133, CD9 and Nestin, and the results showed that the above proteins were decreased significantly after $\mathrm{HBO}$ treatment. This result was consistent with that described by Zeng et al. ${ }^{19}$, who showed that HBO inhibited GBM stemness, with decreased sphere formation and decreased Nestin expression. Hence, according to previous studies and ours, we conclude that because of the decreased expression of 


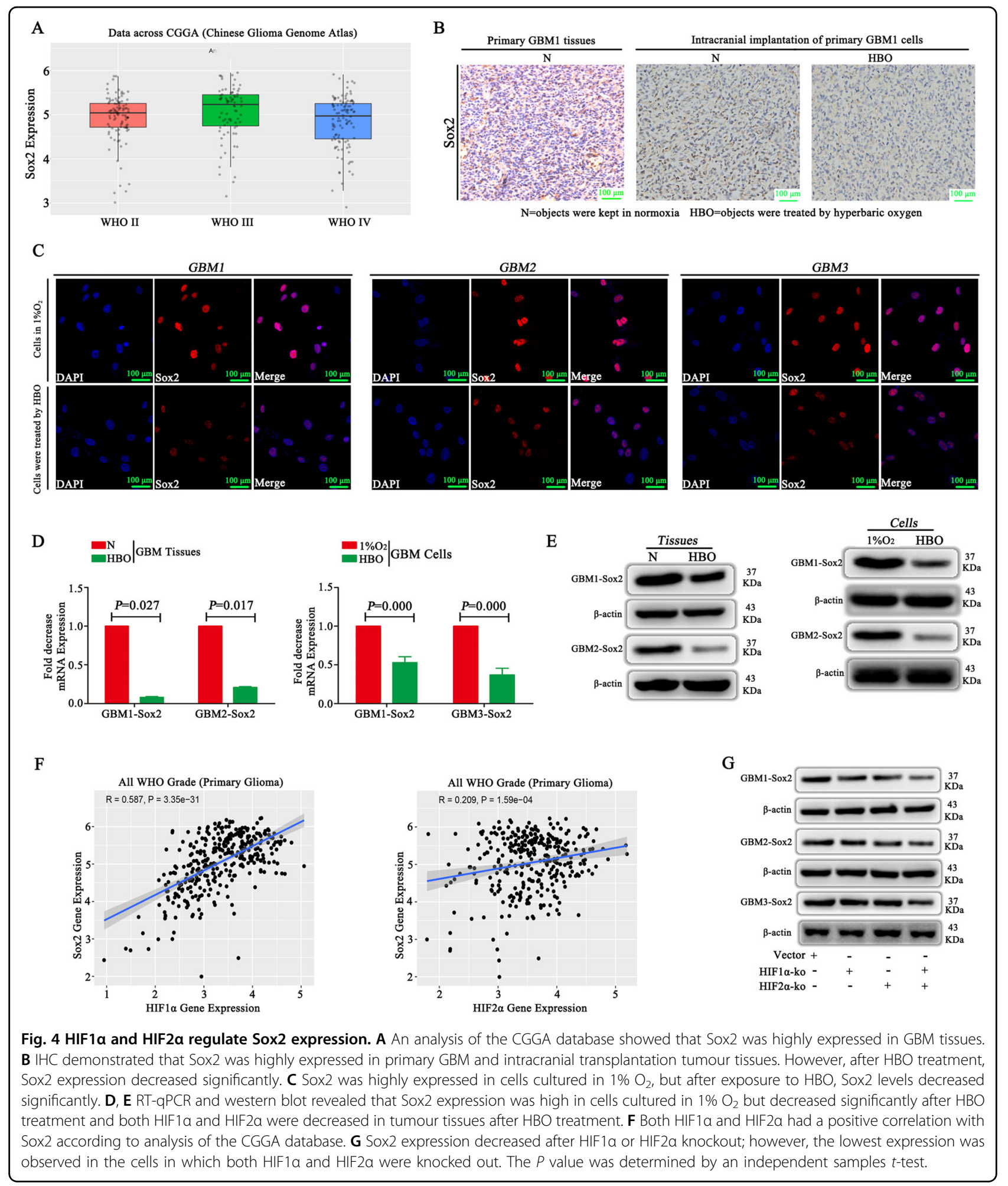

stemness after $\mathrm{HBO}, \mathrm{GBM}$ cells become chemosensitized to TMZ, resulting in a decrease in tumour volume, although tumour volume actually increased under $\mathrm{HBO}$ alone due to cell cycle progression.
The molecular mechanism is also important for understanding the sensitization of GBM cells to chemotherapy under $\mathrm{HBO}$ conditions. To date, only a few studies have reported the mechanism involved and 


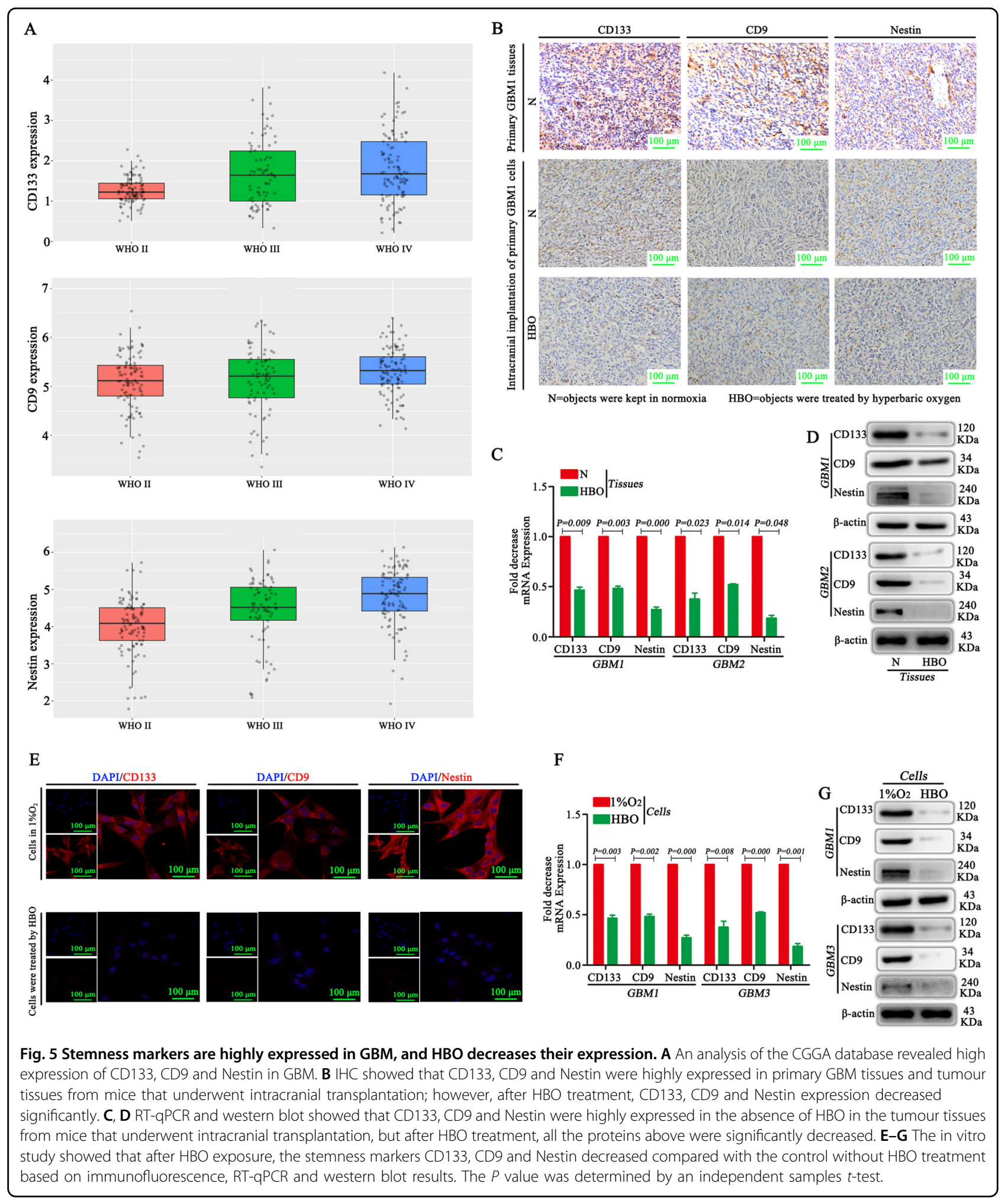

demonstrated that the decrease in TNF- $\alpha$, VEGF and MMP9 contributes to chemosensitization ${ }^{20}$. In contrast to previous studies, we focused on the molecular mechanism through changes in the hypoxic environment. HIF $1 \alpha$ and
HIF2 $\alpha$ are the two main molecules that regulate GBM cell stemness and cell cycle arrest, promoting the resistance of GBM cells to chemotherapy ${ }^{11,21}$. Both HIF1 $\alpha$ and HIF2 $\alpha$ are steadily expressed under hypoxia, but with the 


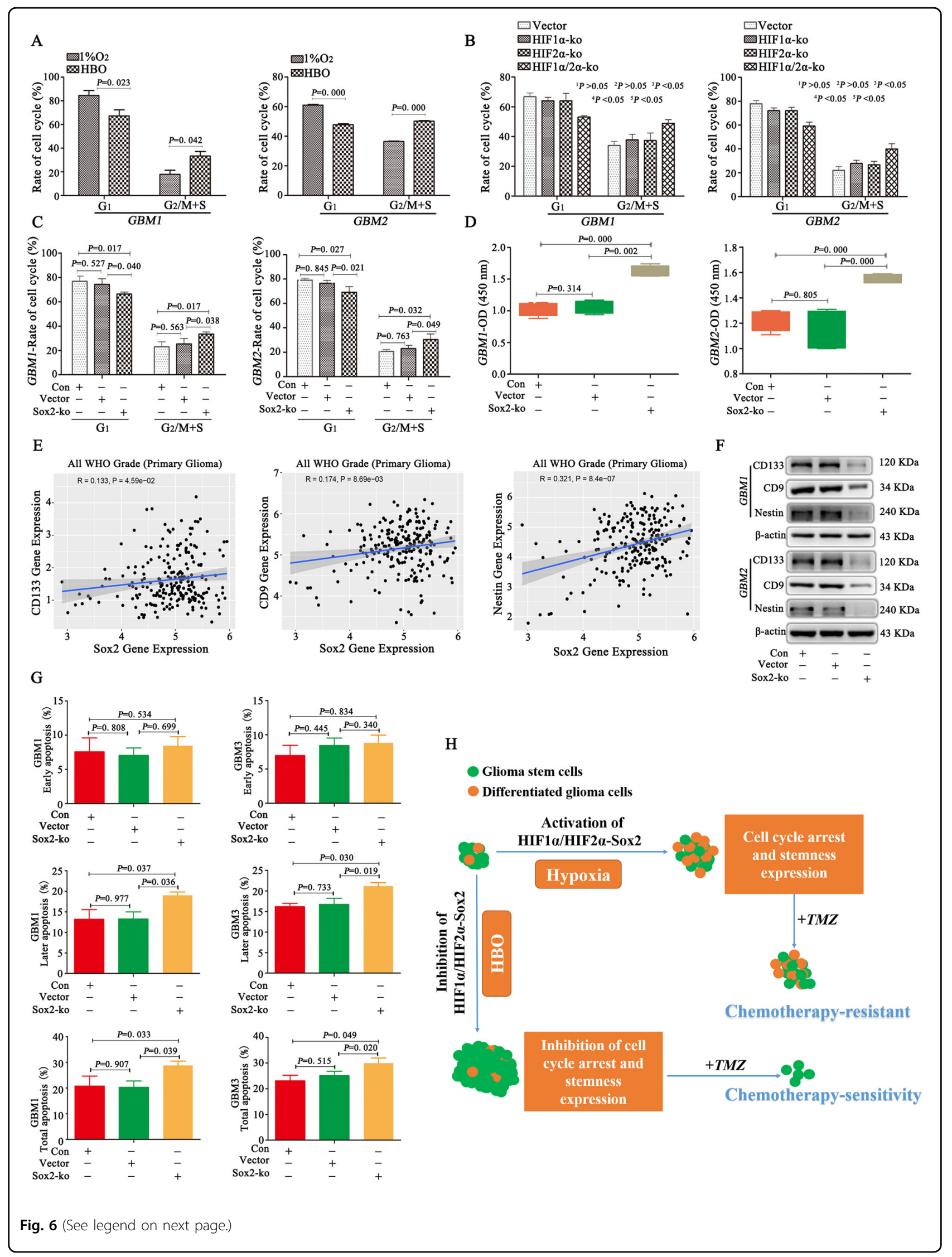


(see figure on previous page)

Fig. $6 \mathrm{HBO}$ increases tumour volume but promotes chemosensitization due to cell cycle progression and decreases stemness by inhibiting HIF1a/HIF2a-Sox2. A GBM cells after HBO exposure had a low proportion of cells in the $\mathrm{G}_{1}$ phase and a high proportion of cells in the $\mathrm{G}_{2} / \mathrm{M}+\mathrm{S}$ phase. $\mathbf{B}$ HIF-ko cells were cultured in $1 \% \mathrm{O}_{2}$ for $72 \mathrm{~h}$, and the proportion of cells in the $\mathrm{G}_{1}$ phase decreased and the proportion of cells in the $G_{2} / M+S$ phase increased after dual HIF1a/HIF2a knockout. Moreover, the proportion of cells in the $G_{1}$ phase was lower and the proportion of cells in the $\mathrm{G}_{2} / \mathrm{M}+\mathrm{S}$ phase was higher in dual HIF1a/HIF2a-KO cells than in individual HIF1a- or HIF2a-KO cells. However, cell cycle changes were not significantly different between the HIF1a- or HIF2a-KO individual group and the empty vector control group. C There were no significant differences between the control and empty vector groups, but there was a decrease in the proportion of cells in the $\mathrm{G}_{1}$ phase and an increase in the proportion of cells in the $G_{2} / M+S$ phase after Sox2 knockout. D After Sox2 knockout in GBM cells, the proliferation rate increased (compared to control and empty vector cells). E A positive correlation was observed between Sox2 and CD133, CD9 and Nestin. F CD133, CD9 and Nestin levels decreased significantly after Sox 2 knockout. G There were much higher late and total apoptosis rates after Sox 2 knockout relative to the control. $\mathbf{H}$ GBM includes glioma stem cells and differentiated glioma cells. When these cells are cultured under hypoxia, HIF1a/HIF2a-Sox2 will become activated, thus promoting cell cycle arrest in the $\mathrm{G}_{1}$ phase and stemness, leading to chemotherapy resistance. However, if these cells are exposed to HBO, HIF1a/ HIF2a-Sox2 will be inhibited, thus inhibiting cell cycle arrest and stemness and promoting not only cell growth but also chemotherapy sensitivity. The $P$ value was determined by an independent samples $t$-test between two groups or one-way ANOVA between at least three groups.

increase in oxygen levels, their expression is decreased significantly ${ }^{11}$. However, to date, only a few reports have described the expression of HIF1 $\alpha$ under HBO conditions in GBM cells, and no studies have reported the influence of $\mathrm{HBO}$ on HIF2 $\alpha$. Concerning the regulatory effect of $\mathrm{HBO}$ on HIF $1 \alpha$, these views remain controversial. The majority of studies have shown that HIF1 $\alpha$ expression in GBM is decreased after $\mathrm{HBO}$ treatment ${ }^{20,22}$, but one article reported that the expression of HIF1 $\alpha$ was increased in HBO-treated glioma tissues compared with control tissues ${ }^{16}$. To clearly determine the influence of HBO on the expression of HIF1 $\alpha$ and HIF2 $\alpha$, we detected their expression in vivo and in vitro and found that both were decreased significantly under $\mathrm{HBO}$ conditions. Hence, we wondered whether the decreased expression of HIF $1 \alpha$ and HIF $2 \alpha$ contributed to the increase in tumour volume and chemosensitization. Through in vitro studies, we surprisingly found that after knocking out HIF1 $\alpha$ or HIF2 $\alpha$ individually, there was no difference in the cell cycle between control and HIF1 $\alpha$ - or HIF $2 \alpha-\mathrm{KO}$ cells. However, we should care that the tumour volume was smaller for HIF1 $\alpha$ - or HIF $2 \alpha-K O$ individual cells in the vivo study, but there was no difference in cell proliferation compared with the control in the in vitro study, which may be due to the short detection time in vitro and the different microenvironments between the in vitro and in vivo studies. After knocking out HIF1 $\alpha$ and HIF2 $\alpha$ at the same time, cell cycle arrest was inhibited, and the proportion of cells in the $G_{2} / M+S$ phase increased, thus contributing to GBM cell proliferation and the increase in tumour volume. In addition, several previous studies have demonstrated that HIF1 $\alpha$ or HIF $2 \alpha$ knockout can inhibit the expression of GBM stemness ${ }^{5,18,23}$. We confirmed that knocking out HIF1 $\alpha$ or HIF $2 \alpha$ decreased the expression of CD133, CD9 and Nestin, especially after knocking out both HIF1 $\alpha$ and HIF2 $\alpha$ at the same time. According to these results, we conclude that $\mathrm{HBO}$ promotes not only GBM growth but also chemosensitization due to the decreased expression of both HIF $1 \alpha$ and HIF $2 \alpha$ by promoting cell cycle progression and inhibiting stemness.

Sox 2 , a transcription factor, is an indispensable element that induces the formation of induced pluripotent stem cells $^{24}$, and their formation ability improves under hypoxic conditions ${ }^{25}$. Previous studies have reported that Sox2 is highly expressed in GBM cells under hypoxia and is considered a marker of stemness ${ }^{12,18,26}$. However, at present, its expression following $\mathrm{HBO}$ exposure is unclear. Therefore, we performed this research and found that Sox2 was not expressed after HBO treatment. Regarding the relationship between HIF1 $\alpha$ and Sox2, Qiang et al. ${ }^{27}$ showed that HIF1 $\alpha$ upregulates Sox2 under hypoxic conditions and promotes stemness. Regarding the HIF2 $\alpha$ and Sox2, a few reports have demonstrated that HIF2 $\alpha$ induces Sox2 in embryonic stem cells ${ }^{28}$, but the correlation between HIF2 $\alpha$ and Sox 2 in GBM is unknown. Therefore, we analysed the relationship using the CGGA database and found that both HIF1 $\alpha$ and HIF2 $\alpha$ exhibited a positive correlation with Sox2. Then, we cultured HIF1 $\alpha$ - and HIF2 $\alpha$-knockout cells under hypoxia and found that Sox 2 expression was decreased and that Sox 2 expression was lowest in dual HIF $1 \alpha / H I F 2 \alpha-K O$ cells. Moreover, we knocked out Sox2 in GBM cells and cultured them in a hypoxic environment. The stem cell markers CD133, Nestin and CD9 were decreased after Sox2 inhibition, which indicates that Sox2 induces stemness in GBM cells under hypoxia. Next, regarding the cell cycle, Otsubo et al. $^{29}$ showed that Sox2 was downregulated in gastric cancers, which led to cell cycle arrest in the $G_{1}$ phase, thus inhibiting cell growth. In contrast, after Sox 2 was overexpressed, the cell cycle progressed and promoted tumour growth. However, some studies have suggested that Sox2 promotes GBM growth as the cell cycle progresses to the $S$ phase ${ }^{13,30}$. In contrast, other studies have suggested that Sox 2 overexpression does not promote GBM cell proliferation, while Sox 2 knockdown induces GBM cell proliferation, thereby increasing cell 
growth $^{31,32}$. Concerning whether Sox2 regulates the cell cycle, we found that Sox2-ko cells had a low proportion of cells in the $G_{1}$ phase but a high proportion of cells in the $\mathrm{G}_{2} / \mathrm{M}+\mathrm{S}$ phase, promoting cell proliferation and $\mathrm{GBM}$ growth according to our results.

In brief, the proposed mechanism is as follows (Fig. 6H). First, HIF $1 \alpha$ and HIF $2 \alpha$ are highly expressed in GBM cells under hypoxia, but the expression of both HIF1 $\alpha$ and HIF2 $\alpha$ is inhibited after HBO treatment. Second, the cell cycle is arrested in the $\mathrm{G}_{1}$ phase under hypoxia, but $\mathrm{HBO}$ promotes cell cycle progression into the $\mathrm{G}_{2} / \mathrm{M}+\mathrm{S}$ phase, thus leading to tumour growth. Third, stemness is active under hypoxia and, as a result, contributes to GBM cell chemoresistance. However, after HBO treatment, stemness is decreased, thus promoting chemosensitization. Fourth, GBM cells grow but become chemosensitive after $\mathrm{HBO}$ treatment due to the progression of the cell cycle but with a decrease in stemness because of the inhibition of HIF $1 \alpha /$ HIF $2 \alpha-S o x 2$. Fifth, not only tumour growth but also chemosensitization after $\mathrm{HBO}$ is ascribed to the downregulation of both HIF $1 \alpha$ and HIF $2 \alpha$ but not the downregulation of HIF $1 \alpha$ or HIF $2 \alpha$ individually. Therefore, according to our results, we conclude that $\mathrm{HBO}$ alone is not suitable for GBM treatment, but the combination of $\mathrm{HBO}$ with chemotherapy is a fine way to cure GBM and improve patient prognosis; the detailed mechanism is attributed to the inhibition of HIF1 $\alpha /$ HIF $2 \alpha$-Sox 2 .

\section{Materials and methods \\ Public data collection}

The Chinese Glioma Genome Atlas (CGGA) database (http://www.cgga.org.cn) was used to analyse the expression of HIF1 $\alpha$, HIF2 $\alpha$, Sox2, CD133, CD9 and Nestin, and correlations among the above proteins was investigated through the dplyr, tibble and ggpolt2 packages in R.

\section{Cell isolation and cell culture}

Primary GBM cells were isolated from tissues obtained from three different patients after surgery and anonymized, and we called them GBM1, GBM2 and GBM3; the detail information is presented in Supplementary Table 5. STR profiling was used to authenticate GBM cells and all the cells were verified none mycoplasma contamination. The tissues were minced and digested at $37^{\circ} \mathrm{C}$ for $45-60$ min with $0.25 \%$ trypsin (HyClone, USA) and $10 \mathrm{U} / \mathrm{ml}$ DNase I (Sigma, USA). ACK lysis buffer (Beyotime Biotechnology, China) was used to lyse red blood cells. Then, the suspension was filtered using a $100-\mu \mathrm{m}$ cell strainer. The obtained cells were cultured in Dulbecco's modified Eagle's medium (DMEM)/F12 (HyClone, USA) supplemented with $10 \%$ foetal bovine serum (FBS, Gibco, USA) to maintain growth in $21 \% \mathrm{O}_{2}$ and $5 \% \mathrm{CO}_{2}$ at $37^{\circ} \mathrm{C}$.

\section{Cell treatment}

Cell growth, cell cycle and protein expression using immunofluorescence and western blotting were detected after cultivation in $1 \% \mathrm{O}_{2}$ for $72 \mathrm{~h}$, and the $\mathrm{HBO}$ group was cultured in $1 \% \mathrm{O}_{2}$ for 3 days but exposed to $\mathrm{HBO}$ for $2 \mathrm{~h}$ each day. mRNA expression was detected after culturing in $1 \% \mathrm{O}_{2}$ for $12 \mathrm{~h}$, and the $\mathrm{HBO}$ group was cultured in $1 \% \mathrm{O}_{2}$ for $12 \mathrm{~h}$ but with $\mathrm{HBO}$ exposure $2 \mathrm{~h}$ before we performed mRNA detection. For cell apoptosis, we added TMZ $(400 \mu \mathrm{M})$ to the medium of GBM cells and cultured it in $1 \% \mathrm{O}_{2}$ for $72 \mathrm{~h}$, and the $\mathrm{HBO}$ group treated with TMZ $(400 \mu \mathrm{M})$ was cultured in $1 \% \mathrm{O}_{2}$ for 3 days but exposed it to $\mathrm{HBO}$ for $2 \mathrm{~h}$ each day. GBM cells were plated into 96 -well plates $\left(4 \times 10^{3}\right.$ cells per well without TMZ, $6 \times 10^{3}$ cells per well with TMZ) with DMEM/F12 $+10 \%$ FBS to detect cell growth using CCK-8. The cell cycle was detected using flow cytometry (FCM), protein expression was detected by immunofluorescence and western blot, and mRNA expression was detected by RTqPCR. Detailed steps of CCK-8, FCM, immunofluorescence, western blotting and RT-qPCR are listed in the Supplementary Materials and Methods.

\section{Immunohistochemistry}

GBM tissues obtained from patients or tumour tissues following intracranial implantation in the absence or presence of HBO were used to detect HIF1 $\alpha$, HIF2 $\alpha$, Sox2, CD133, CD9 and Nestin using IHC. The primary steps of IHC are presented in the Supplementary Materials and Methods.

\section{Hypoxyprobe $^{\mathrm{TM}}-1$ kit}

Anti-human hypoxyprobe ${ }^{\mathrm{TM}}-1$ (Burlington, MA, USA) was intraperitoneally injected $(60 \mathrm{mg} / \mathrm{kg})$, and the mice were fed for an additional $1 \mathrm{~h}$ under normoxia or HBO. Tumour tissues were fixed in $4 \%$ paraformaldehyde, embedded in paraffin, sliced and stained using IHC. For GBM cells, hypoxyprobe ${ }^{\mathrm{TM}}-1(100 \mu \mathrm{mol} / \mathrm{ml})$ was added to the medium, and the cells were cultured for an additional $1 \mathrm{~h}$. Cells were fixed with $4 \%$ paraformaldehyde for $30 \mathrm{~min}$ and stained using immunofluorescence.

\section{HIF and Sox2 knockout assays}

The online CRISPR design programme was used to design plasmid constructs for human HIF $1 \alpha$, HIF $2 \alpha$ and Sox2 single-guide RNAs (sgRNAs). The above sgRNAs (the sequences are listed in Supplementary Table S4) were annealed and cloned into the lentiCRISPRv2 vector (Addgene, \#52961, USA). The lentivirus was transfected into $293 \mathrm{~T}$ cells with the transducing vector, and then the vectors psPAX2 (Addgene \#12260, USA) and pMD2. G were packaged (Addgene \#12259, USA). Forty-eight hours after transfection, the supernatant containing particles was collected, filtered and transduced into GBM cells. 
Western blot analysis was performed to confirm the knockout of HIF1 $\alpha$, HIF $2 \alpha$ and Sox2. The groups were as follows: control, empty vector, HIF $1 \alpha-k o, H I F 2 \alpha-k o$, HIF $1 \alpha / H I F 2 \alpha-k o$ and Sox2-ko. Cell growth, the cell cycle and apoptosis were examined to determine the influence of the downregulation of HIF $1 \alpha$, HIF $2 \alpha$ or Sox 2 on GBM cells from the above groups in the absence or presence of TMZ under $1 \% \mathrm{O}_{2}$ for $72 \mathrm{~h}$. The detailed steps of the above detection are described in the Supplementary Materials and Methods.

\section{In vivo study}

BALB/c-nu mice (male, 4-6 weeks) were used in this study. GBM cells $\left(5 \times 10^{4}\right)$ were seeded into the brains of 100 nude mice, and the mice were randomly divided into four groups: normoxia+DMSO, normoxia+DMSO $+\mathrm{TMZ}, \mathrm{HBO}+\mathrm{DMSO}$ and $\mathrm{HBO}+\mathrm{DMSO}+\mathrm{TMZ}(n=25$ mice per group). TMZ $(2 \mathrm{mg} / \mathrm{kg})$ was injected into the enterocoelia every day from days 3 to 17 in the TMZ treatment groups. $\mathrm{HBO}$ was also administered from days 3 to 17 in the HBO treatment groups. Mice in the TMZ and $\mathrm{HBO}$ groups were injected with $\mathrm{TMZ}(2 \mathrm{mg} / \mathrm{kg})$ within $30 \mathrm{~min}$ after $\mathrm{HBO}$ exposure. $\mathrm{HBO}$ was administered at a pressure of 2.5 ATM for a total of $90 \mathrm{~min}$. Fifteen minutes of pressurization and depressurization were given so that the mice adjusted to changes in pressure. Continuous HBO treatment included a 5-10 min ramp-up to 2.5 ATM under a $100 \% \mathrm{O}_{2}$ environment, followed by sustaining for $90 \mathrm{~min}$ at $2.5 \mathrm{ATM}$, followed by a 10-20 min decompression phase (these steps were performed in accordance with the in vitro study).

Tumour volume was assessed in five mice after feeding for 21 days using MRI. Five mice from each group were sacrificed on day 18 , and tumour tissues were collected to analyse the weight and the expression of HIF1 $\alpha$, HIF $2 \alpha$ and Sox 2 using RT-qPCR, western blot and IHC. The remainder of the mice were used to analyse survival.

Control empty vector cells, HIF1 $\alpha-\mathrm{KO}$ cells, HIF $2 \alpha-\mathrm{KO}$ cells and dual HIF1 $\alpha / \mathrm{HIF} 2 \alpha-\mathrm{KO}$ cells $\left(5 \times 10^{4}\right)$ were injected into the brains of the mice. The mice were randomly divided into eight groups, with 21 mice per group $($ Con+DMSO, Con+DMSO+TMZ, HIF $1 \alpha-k o+D M S O$, HIF $1 \alpha-k o+D M S O+$ TMZ, HIF2 $\alpha-k o+D M S O, H I F 2 \alpha-k o$ + DMSO + TMZ, dual HIF1 $\alpha / \mathrm{HIF} 2 \alpha-k o+$ DMSO, and dual HIF $1 \alpha /$ HIF $2 \alpha-k o+D M S O+$ TMZ) and fed under an environment of $21 \% \mathrm{O}_{2}$. MRI was performed to assess tumour volume on day 21 in five mice. Five mice from each group were sacrificed on day 18 , tumour tissues were collected to analyse the weight, and the remainder of the mice were used to analyse survival. TMZ $(2 \mathrm{mg} / \mathrm{kg})$ was injected into the enterocoelia every day from days 3 to 17 in the TMZ treatment groups.

\section{Statistical analysis}

SPSS 19.0 was used for statistical analysis, and the data are presented as the mean \pm standard deviation (mean \pm $\mathrm{SD})$. Student's $t$-test was used to analyse the significance of differences between two groups, and one-way analysis of variance (one-way ANOVA) was utilized to analyse the significance of differences between at least three groups. The log-rank test was used for survival analysis. Correlations among HIF $1 \alpha$, HIF $2 \alpha$ and Sox 2 were determined according to Pearson's analysis. $P<0.05$ was considered statistically significant.

\section{Author details}

${ }^{1}$ Department of Neurosurgery, Chongqing General Hospital, University of Chinese Academy of Sciences, Chongqing 401147, China. ${ }^{2}$ Chongqing Medical University, Chongqing, China. ${ }^{3}$ Department of Oncology, Chongqing University Cancer Hospital, Chongqing 400030, China

\section{Author contributions}

Designed the experiments, wrote and modified the manuscript: P.W. and N.W.; collected and analysed data: B.L. and S.G.; performed cell cycle and cell apoptosis experiments: B.L. and Q.Y.; performed immunofluorescence and western blot experiments: S.X. and L.Z; RT-qPCR experiments: Y.D.; vivo study: S.G. and J.W.; collected clinical primary glioma tissues: D.Z. and J.P.; performed IHC experiments: J.W.

\section{Funding \\ The National Natural Science Foundation of China (NSFC 81802510) and Yuzhong District project of Chongqing $(20200133,20200134)$ supported this study.}

\section{Conflict of interest}

The authors declare no competing interests.

Ethics statement

The ethics committee of Chongqing General Hospital approved all animal procedures (Ethical code: KY S2021-001-01).

\section{Publisher's note}

Springer Nature remains neutral with regard to jurisdictional claims in published maps and institutional affiliations.

Supplementary information The online version contains supplementary material available at https://doi.org/10.1038/s41420-021-00486-0.

Received: 11 December 2020 Revised: 20 March 2021 Accepted: 16 April 2021

Published online: 13 May 2021

\footnotetext{
References

1. Wen, X. M. et al. Zyxin (ZYX) promotes invasion and acts as a biomarker for aggressive phenotypes of human glioblastoma multiforme. Lab. Invest. 100, 812-823 (2020).

2. Davis, M. E. Glioblastoma: overview of disease and treatment. Clin. J. Oncol. Nurs. 20, S2-S8 (2016).

3. Hegi, M. E. et al. MGMT gene silencing and benefit from temozolomide in glioblastoma. N. Engl. J. Med. 352, 997-1003 (2005).

4. Colwell, N. et al. Hypoxia in the glioblastoma microenvironment: shaping the phenotype of cancer stem-like cells. Neuro-Oncology 19, 887-896 (2017).

5. Li, Z. et al. Hypoxia-inducible factors regulate tumorigenic capacity of glioma stem cells. Cancer Cell 15, 501-513 (2009).
} 
6. Stępień, K., Ostrowski, R. P. \& Matyja, E. Hyperbaric oxygen as an adjunctive therapy in treatment of malignancies, including brain tumours. Med. Oncol. 33, 101 (2016).

7. Wang, Y. G. et al. Hyperbaric oxygen promotes malignant glioma cell growth and inhibits cell apoptosis. Oncol. Lett. 10, 189-195 (2015).

8. Stuhr, L. E. et al. Hyperoxia retards growth and induces apoptosis, changes in vascular density and gene expression in transplanted gliomas in nude rats. $J$. Neuro-Oncol. 85, 191-202 (2007).

9. Xie, Y. et al. Hyperbaric oxygen as an adjuvant to temozolomide nanoparticle inhibits glioma growth by inducing G2/M phase arrest. Nanomedicine (Lond.) 13, 887-898 (2018)

10. Dagistan, Y. et al. Combination hyperbaric oxygen and temozolomide therapy in C6 rat glioma model. Acta Cir. Bras. 27, 383-387 (2012).

11. Keith, B., Johnson, R. S. \& Simon, M. C. HIF1a and HIF2a: sibling rivalry in hypoxic tumour growth and progression. Nat. Rev. Cancer 12, 9-22 (2011).

12. Wang, J. et al. Invasion of white matter tracts by glioma stem cells is regulated by a NOTCH1-SOX2 positive-feedback loop. Nat. Neurosci. 22, 91-105 (2019).

13. Mamun, M. A., Mannoor, K., Cao, J., Qadri, F. \& Song, X. SOX2 in cancer stemness: tumour malignancy and therapeutic potentials. J. Mol. Cell Biol. 12 85-98 (2020).

14. Wang, P., Wan, W. W., Xiong, S. L., Feng, H. \& Wu, N. Cancer stem-like cells can be induced through dedifferentiation under hypoxic conditions in glioma, hepatoma and lung cancer. Cell Death Discov. 3, 16105 (2017).

15. Ahmed, E. M., Bandopadhyay, G., Coyle, B. \& Grabowska, A. A HIF-independent, CD133-mediated mechanism of cisplatin resistance in glioblastoma cells. Cell. Oncol. (Dordr.) 41, 319-328 (2018).

16. Ding, J. B., Chen, J. R., Xu, H. Z. \& QinZ, Y. Effect of hyperbaric oxygen on the growth of intracranial glioma in rats. Chin. Med. J. 128, 3197-3203 (2015).

17. Wang, P. et al. HIF1a regulates glioma chemosensitivity through the transformation between differentiation and dedifferentiation in various oxygen levels. Sci. Rep. 7, 7965 (2017).

18. Wang, P. et al. HIF1a regulates single differentiated glioma cell dedifferentiation to stem-like cell phenotypes with high tumorigenic potential under hypoxia. Oncotarget 8, 28074-28092 (2017).
19. Zeng, X. F. et al. Mild thermotherapy and hyperbaric oxygen enhance sensitivity of TMZ/PSi nanoparticles via decreasing the stemness in glioma. J. Nanobiotechnol. 17, 47 (2019).

20. Lu, Z. H. et al. Hyperbaric oxygen therapy sensitizes nimustine treatment for glioma in mice. Cancer Med. 5, 3147-3155 (2016).

21. Young, R. M. \& Simon, M. C. Untuning the tumor metabolic machine: HIF-a: pro- and antitumorigenic? Nat. Med. 18, 1024-1025 (2012).

22. Zembrzuska, K., Ostrowski, R. P. \& Matyja, E. Hyperbaric oxygen increases glioma cell sensitivity to antitumor treatment with a novel isothiourea derivative in vitro. Oncol. Rep. 41, 2703-2716 (2019).

23. Nusblat, L. M., Tanna, S. \& Roth, C. M. Gene silencing of HIF-2a disrupts glioblastoma stem cell phenotype. Cancer Drug Resist. 3, 199-208 (2020).

24. Takahashi, K. et al. Induction of pluripotent stem cells from adult human fibroblasts by defined factors. Cell 131, 861-872 (2007).

25. Mathieu, J. et al. HIF induces human embryonic stem cell markers in cancer cells. Cancer Res. 71, 4640-4652 (2011).

26. Hattermann, K. et al. Stem cell markers in glioma progression and recurrence. Int. J. Oncol. 49, 1899-1910 (2016).

27. Qiang, L. et al. HIF-1a is critical for hypoxia-mediated maintenance of glioblastoma stem cells by activating Notch signaling pathway. Cell Death Differ. 19, 284-294 (2012).

28. Forristal, C. E., Wright, K. L., Hanley, N. A., Oreffo, R. O. \& Houghton, F. D. Hypoxia inducible factors regulate pluripotency and proliferation in human embryonic stem cells cultured at reduced oxygen tensions. Reproduction 139, 85-97 (2010).

29. Otsubo, T., Akiyama, Y., Yanagihara, K. \& Yuasa, Y. SOX2 is frequently downregulated in gastric cancers and inhibits cell growth through cell-cycle arrest and apoptosis. Br. J. Cancer 98, 824-831 (2008).

30. Oppel, F. et al. SOX2-RNAi attenuates S-phase entry and induces RhoAdependent switch to protease-independent amoeboid migration in human glioma cells. Mol. Cancer 10, 137 (2011).

31. Weina, K. \& Utikal, J. SOX2 and cancer: current research and its implications in the clinic. Clin. Transl. Med. 3, 19 (2014).

32. Wuebben, E. L. \& Rizzino, A. The dark side of SOX2: cancer-a comprehensive overview. Oncotarget 8, 4917-44943 (2017). 